\title{
The Surgical Treatment of Irradiated Wounds: A Report on 36 Patients
}

\author{
Akira Saito $^{1}$, Noriko Saito ${ }^{2}$, Emi Funayama ${ }^{2}$ and Hidehiko Minakawa ${ }^{1}$ \\ ${ }^{1}$ Division of Plastic and Reconstructive Surgery, Hokkaido Cancer Center, Sapporo, Japan \\ ${ }^{2}$ Department of Plastic and Reconstructive Surgery, Graduate School of Medicine, Hokkaido University, \\ Sapporo, Japan
}

Received 10 December 2012; Accepted 24 December 2012; Published 9 January 2013

Academic Editor: Hisashi Watanabe

\begin{abstract}
Radiation therapy, although a useful modality for cancer therapy, injures the surrounding uninvolved tissues. Although surgery is often necessary for non-healing ulcers, surgery of irradiated wounds is recognized as challenging. In this study, we evaluate the outcomes and complications of patients who underwent surgical treatment of irradiated wounds. We reviewed 36 patients who underwent reconstructive surgery for irradiated wounds at our institution. A regional flap was applied in 17 patients, a local flap in 13 patients, a skin graft in 2 patients, and direct closure was performed in 4 patients. The complication rate was $65 \%, 69 \%, 50 \%$, and $25 \%$, respectively, and was not significantly different based on the type of reconstructive procedure used. Of the 30 patients who underwent flap reconstruction, 21 (70\%) had flaps that included muscle and the remaining 9 patients (30\%) had flaps that did not include muscle. The complication rate was $67 \%$ for both groups. Thus, the use of a muscle flap did not lead to superior outcomes. A radiation dose $>60$ Gy was correlated with a higher wound complication rate $(p<0.001)$. This study demonstrated that the use of regional flaps did not diminish the complication rate; moreover, contrary to previous reports, the use of muscle/musculocutaneous flaps did not reduce complications. Radiotherapy dose was the only factor associated with wound complications. Although reconstructive surgery of wounds irradiated with high-dose radiation was quite difficult, most of the irradiated wounds in our study were ultimately closed.
\end{abstract}

Keywords: Irradiated wounds; radiation ulcer; radiotherapy; reconstruction.

\section{Introduction}

Radiation therapy is important for the management of malignant tumors. However, the deleterious effects of radiation, both immediate and long-term, may have a significant impact on local tissues. Although surgical attempts at wound closure are often necessary for non-healing ulcers that may develop at any time following radiotherapy (Goessler, Bugert, Kassner, Stern-Straeter, Bran, , Sadick, Hormann \& Riedel, 2010), surgery of irradiated wounds is recognized as challenging (Arnold, Lovich \& Pairolero,

Copyright (C) 2013 Akira Saito, Noriko Saito, Emi Funayama and Hidehiko Minakawa. This is an open access article distributed under the Creative Commons Attribution License unported 3.0, which permits unrestricted use, distribution, and reproduction in any medium, provided that original work is properly cited. Contact author: Akira Saito E-mail: hmsaxena@yahoo.com 
1994). The objective of this study was to evaluate the outcomes and complications of 36 patients who underwent surgical treatment of irradiated wounds.

\section{The Main Body of the Paper}

\section{Patients and Methods}

A retrospective analysis of patients who underwent reconstructive surgery for irradiated wounds at the Division of Plastic and Reconstructive Surgery, Hokkaido Cancer Center, from 2001 to 2012 was performed. There were 18 men and 18 women, with a median age of 66 years (range 25-83 years). The lesions were located on the head and neck in 12 patients, the trunk in 16 patients, and on the extremities in 8 patients.

For patients with head and neck wounds, the primary diseases included laryngeal cancer $(\mathrm{n}=4)$, tongue cancer $(\mathrm{n}=2)$, hypopharyngeal cancer $(n=2)$, skin cancer $(n=1)$, brain tumor $(n=1)$, parotid cancer $(n$ $=1$ ), and a metastatic brain tumor $(\mathrm{n}=1)$. For the patients with trunk wounds, sarcoma $(n=5)$, metastatic bone tumor $(n=5)$, breast cancer ( $n=4)$, lung cancer $(n=1)$, and double cancer of the lung and breast $(n=1)$ were the primary diseases. For patients with wounds on the extremities, sarcoma $(n=6)$, skin cancer $(\mathrm{n}=1)$, and metastatic bone tumor $(n=1)$ were the original diseases.
Thirty-two patients had previously undergone 1 or more surgical interventions for the original disease, and 4 patients had not undergone any such surgical intervention. All of the patients were treated with radiation therapy: 33 received external radiation therapy; 1 received implant radiation therapy; and 2 received external radiation therapy and implant radiation therapy. The mean dose of radiotherapy was $58.5 \mathrm{~Gy}$. The mean time elapsed from radiation to wound onset was 6 years and 6 months.

We also studied the impact of the following factors on post-operative complications: age; recipient site; prior surgery; radiation dose; time from radiation to wound onset; comorbidity (diabetes); type of reconstruction; and type of flap. We compared each of these factors with the presence of post-operative wound complications and wound recurrence. Statistical differences between categorical variables were determined using Chi-square or Fisher's exact probability tests. Test results with $\mathrm{p}<0.05$ were regarded as significant.

\section{Results}

Reconstructive procedures based on the site of the lesion are shown in Table 1.

Table 1. Site of Lesion and Reconstructive Options

\begin{tabular}{|l|l|c|c|c|c|c|}
\hline & & Suture & Skin graft & Local flap & Regional flap & Total \\
\hline & Head and neck & 3 & 0 & 3 & 6 & 12 \\
\hline & Trunk & 1 & 1 & 6 & 8 & 16 \\
\hline & Extremities & 0 & 1 & 4 & 3 & 8 \\
\hline & Total & 4 & 2 & 13 & 17 & 36 \\
\hline
\end{tabular}

Regional flaps were the most commonly applied (47\%), followed by local flaps (36\%). In total, 30 patients underwent flap surgery. Table 2 shows the various types of flaps used based on the site of the lesion. Musculocutaneous flaps were the most frequently utilized flap type (57\%), followed by cutaneous flaps (20\%). Twenty-one patients $(70 \%)$ had flaps that included muscle (muscle or musculocutaneous flaps) and the remaining 9 patients (30\%) had flaps that did not include muscle (cutaneous, fasciocutaneous, or mucosal flaps). 
Table 2. Site of Lesion and Flap Type

\begin{tabular}{|l|l|c|c|c|c|c|c|}
\hline & $\begin{array}{c}\text { Mucosal } \\
\text { flap }\end{array}$ & $\begin{array}{c}\text { Cutaneous } \\
\text { flap }\end{array}$ & FC flap & MC flap & $\begin{array}{c}\text { Muscle } \\
\text { flap }\end{array}$ & Total \\
\hline & Head and neck & 1 & 2 & 1 & 4 & 1 & 9 \\
\hline Trunk & 0 & 2 & 1 & 9 & 2 & 14 \\
\hline & Extremities & 0 & 2 & 0 & 4 & 1 & 7 \\
\hline & Total & 1 & 6 & 2 & 17 & 4 & 30 \\
\hline & & & & & & \\
\hline & FC flap: fasciocutaneous flap, MC flap: musculocutaneous flap & & & \\
\hline
\end{tabular}

Among all patients, 22 (61\%) developed post-operative complications and 20 (56\%) of these had wound recurrence. Table 3 shows the complications at the recipient site. The most commonly encountered complication was wound dehiscence (19\%) followed by infection (13\%) and partial flap loss (11\%). Of the 22 patients who experienced complications, 9 required surgical revision while the others were treated conservatively. Of the 9 patients who underwent revision surgery, 5 underwent an additional flap surgery (4 local flaps and 1 regional flap).

Table 3. Complications at the Recipient Site

\begin{tabular}{|c|l|c|}
\hline & Complications & Number \\
\hline & Wound dehiscence & 7 \\
\hline & Infection & 5 \\
\hline & Partial flap necrosis & 4 \\
\hline & Necrosis of original skin & 2 \\
\hline & Recurrence of fistulae & 2 \\
\hline & Fistulae & 1 \\
\hline & Hematoma & 1 \\
\hline & Incomplete graft necrosis & 1 \\
\hline
\end{tabular}

Table 4 summarizes the wound morbidity results for each factor. A radiation dose $>60$ Gy was correlated with a higher wound complication rate $(p<0.001)$ and wound recurrence $(\mathrm{p}<0.0001)$. No other factor was associated with post-operative wound complications or wound recurrence. 
Table 4. Effect of Categorical Variables on Wound Complications

\begin{tabular}{|c|c|c|c|c|c|c|c|}
\hline \multirow[t]{2}{*}{ Variables } & & \multicolumn{2}{|c|}{ Complication } & \multirow[t]{2}{*}{$\mathrm{P}$} & \multicolumn{2}{|c|}{$\begin{array}{l}\text { Wound } \\
\text { recurrence }\end{array}$} & \multirow[t]{2}{*}{$\mathrm{P}$} \\
\hline & & yes & no & & yes & no & \\
\hline \multicolumn{8}{|l|}{ Age } \\
\hline & $<70$ years & 14 & 9 & 0.96 & 12 & 11 & 0.58 \\
\hline & $>70$ years & 8 & 5 & & 8 & 5 & \\
\hline \multicolumn{8}{|l|}{ Site } \\
\hline & Head and neck & 8 & 4 & 0.74 & 8 & 4 & 0.43 \\
\hline & Trunk & 10 & 6 & & 9 & 7 & \\
\hline & Extremities & 4 & 4 & & 3 & 5 & \\
\hline \multicolumn{8}{|c|}{ Prior surgery } \\
\hline & Yes & 18 & 14 & 0.09 & 16 & 16 & 0.057 \\
\hline & No & 4 & 0 & & 4 & 0 & \\
\hline \multicolumn{8}{|c|}{ Radiation dose } \\
\hline & $<60 \mathrm{~Gy}$ & 6 & 10 & $<0.001$ & 4 & 12 & $<0.0001$ \\
\hline & $>60 \mathrm{~Gy}$ & 15 & 1 & & 15 & 1 & \\
\hline \multicolumn{8}{|c|}{ Time from radiotherapy to wound onset } \\
\hline & $<6$ months & 7 & 5 & 0.96 & 6 & 6 & 0.84 \\
\hline & $>6$ months, $<2$ years & 7 & 4 & & 6 & 5 & \\
\hline & $>2$ years & 8 & 5 & & 8 & 5 & \\
\hline \multicolumn{8}{|l|}{ DM } \\
\hline & Yes & 5 & 3 & 0.92 & 5 & 3 & 0.65 \\
\hline & No & 17 & 11 & & 15 & 13 & \\
\hline \multicolumn{8}{|c|}{ Reconstructive procedure } \\
\hline & suture & 1 & 3 & 0.43 & 1 & 3 & 0.61 \\
\hline & skin graft & 1 & 1 & & 1 & 1 & \\
\hline & local flap & 9 & 4 & & 8 & 5 & \\
\hline & regional flap & 11 & 6 & & 10 & 7 & \\
\hline \multicolumn{8}{|c|}{ Muscle included in flap } \\
\hline & Yes & 6 & 3 & 1.00 & 6 & 3 & 0.62 \\
\hline & No & 14 & 7 & & 12 & 9 & \\
\hline
\end{tabular}

Despite the high complication rate experienced with all techniques, most of the irradiated wounds were ultimately closed. Among the 35 patients, 31 (89\%) had irradiated wounds that had healed at last follow up, and only 4 (11\%) had irradiated wounds that had not healed. It took 104.9day on average (range 21-421days) for healing in the patients with wound recurrence.

\section{Discussion}

Radiation therapy, although a useful modality for cancer therapy, injures the surrounding uninvolved tissues (Goessler et al., 2010; Dormand, Banwell \& Goodacre, 2005). Tissue damaged by radiation may result in chronic, painful, and poorly healing ulcers. Increased difficulty healing after surgery and wound 
recurrence may also occur even with minor trauma (Vivas, Tang, Escandon \& Kirsner, 2011). Irradiated wounds are often resistant to conventional treatment modalities (Vivas et al., 2011), and may often require surgical reconstructive intervention. However, irradiated wounds continue to be a vexing therapeutic challenge for reconstructive surgeons (Riedel, Philipp, Sadick, Goessler, Hormann \& Verse, 2005).

The principle of surgical management of the irradiated field is generous debridement and coverage of the defect with well-vascularized non-irradiated tissue (Kurul, Dincer, Kizir, Uzunismail \& Darendeliler, 1997; Samuels, Granick, Ramasastry, Solomon \& Hurwitz, 1993; Aslan, Terzioglu, Tuncali \& Bingul, 2004). The choice of reconstructive procedure depends on the site of the lesion, the size of the defect, age, the general condition of the patient, and the local condition of the lesion (Brenner, Reichert, Schafers, Lister, Dreyer \& Berger, 1995). The reconstructive options usually include skin grafts, local flaps, regional flaps, or free flaps.

Skin grafts may be unsuitable for patients with chronic radiation wounds because of impairment of the vascularity of the recipient bed (Kurul et al., 1997). Rudolph (1982) reported that thin split-thickness skin grafts for ulcer treatment had a 100\% complication rate. Kurul et al. (1997) investigated the outcomes of 200 previously irradiated patients who underwent skin grafting or flap reconstruction. They reported that the complication rate with skin grafts was significantly higher than that with skin or muscle flaps.

Local flaps are often unsuitable as the surrounding areas have usually been affected by irradiation and are thus inelastic and unreliable (Robinson, 1975). Rudolph (1982) reported that local flaps had a high complication rate even if the flaps were delayed. The complication rate was $100 \%$ in the patients with delayed flaps and $88 \%$ among those with non-delayed flaps. Arnold et al. (1994) investigated outcomes with muscle flaps rotated from irradiated areas and reported that because of the high complication rate, the use of regional muscle flaps was preferable. Compared to skin grafts and local flaps, regional flaps with uninvolved tissue derived from outside the irradiated field are possibly a good choice (Samuels et al., 1993; Di Meo \& Jones ,1984).

In the present study, we applied a regional flap in 17 patients, a local flap in 13 patients, skin grafts in 2 patients, and we performed direct wound closure in 4 patients. The complication rate was 65\% (11 patients), $69 \%$ (9 patients), 50\% (1 patient), and 25\% (1 patient), respectively. The complication rate with regional flaps was just as high as that with local flaps, and was not significantly different when analyzed according to the type of reconstructive procedure performed.

The use of muscle and musculocutaneous flaps for the reconstruction of defects in irradiated areas has been well described in the literature as these are more resistant to bacterial inoculation, more prone to clean residual infection, better vascularized, and more voluminous and thus better for filling contour defects (Arnold et al.,1994; Dormand et al., 2005; Vivas et al., 2011; Kurul, et al., 1997). Arnold et al. (1994) reported that the complication rate was $19 \%$ when nonirradiated muscle flaps were used. Kurul et al. (1997) reported that the complication rate was as low as $6 \%$ when muscle/musculocutaneous flaps were used, although there were no significant differences in complication rate between patients with skin and muscle flaps. In the present study, of the 30 patients who underwent flap reconstruction, 21 (70\%) had flaps that included muscle (muscle or musculocutaneous flaps) and the remaining 9 patients $(30 \%)$ had flaps that did not include muscle (cutaneous, fasciocutaneous, or mucosal flaps). The complication rate was $67 \%$ in both groups. Thus, we did not find that the use of muscle and musculocutaneous flaps led to superior outcomes. 
Although the causes of the complications at the recipient site are likely to be multifactorial, insufficient debridement appears to be one responsible factor since most of the complications that developed were likely to be attributed to insufficient debridement: 7 cases of wound dehiscence; 2 cases of fistula recurrence; 1 case of fistula development; 2 cases of necrosis of the original skin; and 1 case of incomplete graft necrosis. We attempted to debride the irradiated wound when consideration of clinical parameters such as skin characteristics and bleeding from the tissue margins indicated that this was feasible. However, it was difficult to precisely determine the areas affected.

There have been few reports on the impact of reconstructive surgery of irradiated areas on post-operative complications (Kurul, et al., 1997). In our series, radiotherapy dose (>60 Gy) was the only factor associated with wound complications. The patients frequently had received high doses of radiation ( $>60 \mathrm{~Gy}$ ) as radical radiotherapy in our center. All 4 patients who had irradiated wounds that were unhealed at last follow up had received radiotherapy with doses $>60 \mathrm{~Gy}$. After high doses of radiation, the irradiated areas might be more deeply damaged and so the original estimates of the area involved might have been vastly underestimated, which meant that we could not achieve adequate debridement despite aggressive attempts to do so. As a result of insufficient debridement, complications might have developed post-operatively in many patients. Special care is necessary when planning and performing reconstructive surgery for wounds irradiated with high-dose radiation therapy.

In conclusion, this study demonstrates that the use of regional flaps did not diminish the post-operative complication rate; moreover, the use of muscle/musculocutaneous flaps did not reduce the incidence of postoperative complications, which is contrary to previous reports. We assume that adequate debridement is critical for the treatment of irradiated wounds, and postoperative complication would occur as a result of insufficient debridement even if muscle/musculocutaneous flaps were employed. Radiotherapy dose ( $>60 \mathrm{~Gy}$ ) was the only factor associated with wound complications. Although reconstructive surgery of wounds irradiated with high-dose radiation was quite difficult, most of the irradiated wounds were ultimately closed.

\section{References}

Arnold, P. G., Lovich, S. F. \& Pairolero, P. C. (1994). "Muscle Flaps in Irradiated Wounds: An Account of 100 Consecutive Cases," Plastic and reconstructive surgery, 93 (2) 324-327; discussion 328-329.

Aslan, G., Terzioglu, A., Tuncali, D. \& Bingul, F. (2004). "Consequences of Radiation Accidents," Annals of plastic surgery, 52 (3) 325-328.

Brenner, P., Reichert, B., Schafers, H.- J., Lister, G. D., Dreyer, J. O. \& Berger, A. (1995). "Chest Wall Reconstruction by Free Latissimus Dorsi Flap 25 Years after Accidental Exposure to a Source of Industrial Irradiation," British journal of plastic surgery, 48 (6) 431-433.

Di Meo, L. \& Jones, B. M. (1984). "Surgical Treatment of Radiation-Induced Scalp Lesions," British journal of plastic surgery, 37 (3) 373-378.

Dormand, E. L., Banwell, P. E. \& Goodacre, T. E. (2005). "Radiotherapy and Wound Healing," International wound journal, 2 (2) 112-127.

Goessler, U. R., Bugert, P., Kassner, S., SternStraeter, J., Bran, G., Sadick, H., Hormann, K. \& Riedel, F. (2010). "In Vitro Analysis of Radiation-Induced Dermal Wounds," Otolaryngology--head and neck surgery, 142 (6) 845-850. 
7 Plastic Surgery: An International Journal

Kurul, S., Dincer, M., Kizir, A., Uzunismail, A. \& Darendeliler, E. (1997). "Plastic Surgery in Irradiated Areas: Analysis of 200 Consecutive Cases," European journal of surgical oncology, 23 (1) 48-53.

Riedel, F., Philipp, K., Sadick, H., Goessler, U., Hormann, K. \& Verse, T. (2005). "Immunohistochemical Analysis of Radiation-Induced Non-Healing Dermal Wounds of the Head and Neck," In Vivo, 19 (2) 343-350.

Robinson, D. W. (1975). "Surgical Problems in the Excision and Repair of Radiated Tissue," Plastic and reconstructive surgery, 55 (1) 41-49.

Rudolph, R. (1982). "Complications of Surgery for Radiotherapy Skin Damage," Plastic and reconstructive surgery, 70 (2), 179-185.

Samuels, L., Granick, M. S., Ramasastry, S., Solomon, M. P. \& Hurwitz, D. (1993). "Reconstruction of Radiation-Induced Chest Wall Lesions," Annals of plastic surgery, 31 (5) 399-405.

Vivas, A., Tang, J. C., Escandon, J. \& Kirsner, R. S. (2011). "Postradiation Chronic Scalp Ulcer: A Challenge for Wound Healing Experts," Dermatologic surgery, 37 (11) 1693-1696. 